\title{
Internal rapid rotation and its implications for stellar structure and pulsations
}

\author{
Daniel R. Reese ${ }^{1,2, a}$ \\ 1 School of Physics and Astronomy, University of Birmingham, Edgbaston, Birmingham, B15 2TT, \\ UK \\ 2 Stellar Astrophysics Centre (SAC), Department of Physics and Astronomy, Aarhus University, Ny \\ Munkegade 120, DK-8000 Aarhus C, Denmark
}

\begin{abstract}
Massive and intermediate mass stars play a crucial role in astrophysics. Indeed, massive stars are the main producers of heavy elements, explode in supernovae at the end of their short lifetimes, and may be the progenitors of gamma ray bursts. Intermediate mass stars, although not destined to explode in supernovae, display similar phenomena, are much more numerous, and have some of the richest pulsation spectra. A key to understanding these stars is understanding the effects of rapid rotation on their structure and evolution. These effects include centrifugal deformation and gravity darkening which can be observed immediately, and long terms effects such as rotational mixing due to shear turbulence, which prolong stellar lifetime, modify chemical yields, and impact the stellar remnant at the end of their lifetime. In order to understand these effects, a number of models have been and are being developed over the past few years. These models lead to increasingly sophisticated predictions which need to be tested through observations. A particularly promising source of constraints is seismic observations as these may potentially lead to detailed information on their internal structure. However, before extracting such information, a number of theoretical and observational hurdles need to be overcome, not least of which is mode identification. The present proceedings describe recent progress in modelling these stars and show how an improved understanding of their pulsations, namely frequency patterns, mode visibilities, line profile variations, and mode excitation, may help with deciphering seismic observations.
\end{abstract}

\section{Introduction}

\begin{abstract}
Massive stars play an important role in astrophysics. Indeed, they are the main producers of heavy elements, dominate the chemical evolution of galaxies, and end their lives in supernovae. Although intermediate mass stars do not explode in supernovae, they present many of the same phenomena as massive stars, and furthermore can have very rich pulsation spectra. Therefore, understanding the structure and evolution of these stars, in particular their chemical evolution and internal transport processes, is crucial. Unlike their less massive counterparts, these stars hardly have any surface convection (apart from possibly a thin near-surface convective layer). Consequently, they are not spun down and tend to be rapid rotators (e.g. [1]). Hence, in order to understand these these stars, one needs to understand the impact of rapid rotation on stellar structure, chemical evolution, and pulsations.
\end{abstract}

\footnotetext{
a e-mail: dreese@bison.ph.bham.ac.uk
} 


\section{Impact of rotation on stellar structure and evolution}

Rapid stellar rotation has both immediate effects on stellar structure as well as long range effects on stellar evolution.

\subsection{Structural changes}

One of the first and most obvious effects of rapid rotation is stellar deformation. Indeed, the centrifugal force causes the equator to bulge, as confirmed by recent interferometric observations (e.g. [2], [3], [4]). This effect is important because it means these stars can no longer be modelled using a 1D approach but require a $2 \mathrm{D}$ approach.

A second effect, which also has immediate consequences, is gravity darkening. Indeed, in rapidly rotating stars, the equator is less luminous than the poles, due to a smaller vertical temperature gradient. This effect has also been observed through interferometry (e.g. [5], [6], [7]) and is important because it causes the position of these stars in an HR diagram to depend on their inclination (e.g. [8], [9]).

Various approaches have been used to model this effect, the first being von Zeipel's law [10]. This approach corresponds to the simple power law $T_{\mathrm{eff}} \propto g_{\mathrm{eff}}^{\beta}$, where $\beta=0.25$. The value $\beta=0.8$ has also been proposed for stars with a convective envelope [11]. More recently, Espinosa Lara and Rieutord came up with a new law which is based on two assumptions: first, that the luminous flux is parallel to the effective gravity, and second that the divergence of the luminous flux is zero (which corresponds to assuming no production of energy in the envelope). This leads to an analytical solution which does not depend on any free parameters, and which compares very favourably with full $2 \mathrm{D}$ numerical simulations from the ESTER code [12].

\subsection{Baroclinic effects}

As opposed to their non-rotating counterparts, rapidly rotating stars have a baroclinic structure. In other words, lines of constant pressure, temperature, and density no longer coincide. This produces differential rotation which in turn leads to meridional circulation due to viscosity [13]. Currently, only the ESTER code is able to self-consistently calculate such flows in realistic 2D stellar models ([14], [15]).

As a result of various instabilities, baroclinic flows will then cause turbulence and enhance transport processes, which modify the stellar lifetime and chemical yields (e.g. [16]). Predictions from 1D stellar evolution codes including a shellular rotation profile based on [17] are in better agreement with various observations, such as the chemical enrichment of OBA stars, the number ratio of red to blue supergiants etc. (see [18] and references therein). Recent observations of $\mathrm{N}$ enrichment in $\mathrm{O}$ and $\mathrm{B}$ stars ([19], [20]) have, however, shown discrepancies with theoretical expectations. Indeed, there is a lack of correlation between $\mathrm{N}$ enrichment and equatorial velocity, whereas rotational mixing is needed in theoretical models to produce $\mathrm{N}$ enrichment. It is not clear what could cause this, although various solutions have been proposed, such as transport of chemical species by waves [21].

\subsection{Impact on convection zones}

Rapid rotation also affects convection zones. For instance, [22] produced a rapidly rotating model with a convectively unstable equatorial belt. A year later, [23], using a 1D formalism, found that rotation favours convection in stellar envelopes, especially at the equator. Nonetheless, it remains an open question as to what prescription for convection should be used in a 2D rapidly rotating model, and is currently the main reason why the ESTER code is unable to model low mass stars. However, ongoing work by [24] may provide an answer to this question. Indeed, they are developing a new convection code based on unstructured meshes, capable of handling centrifugal deformation. Initial comparisons with benchmarks from the ASH code show promising results. 


\subsection{Summary}

In summary, rotation causes many new phenomena which affect stellar structure, transport processes, mixing, and evolution. Although much progress has been made in our theoretical modelling of these processes, there remains large uncertainties due to their complexity and to discrepancies with current observations. As a result, it is necessary to constrain these processes through further observations, and asteroseismology is currently the best way of doing this as it is the only way we have to probe the internal structure of stars. However, before being able to carry out asteroseismology, one needs to be able to model the effects of rapid rotation on stellar pulsations, which, as will be described in the next section, is by no means trivial.

\section{Impact of rotation on stellar pulsations}

In the non-rotating case and in the absence of phenomena which break spherical symmetry, such as magnetic fields, stellar pulsation modes with the same radial order, $n$, and harmonic degree, and $\ell$, but differing azimuthal orders, $m$, have the same frequencies and are thus degenerate. However, it has been known for many years that rotation lifts this degeneracy [25], in much the same way that the Zeeman effect splits absorption lines. The first order effect of rotation can be split into two terms. The first is simply an advection term which leads to a sort of Doppler shift of the frequencies on non-axisymmetric modes. The second comes from the effects of the Coriolis force and is characterised by the Ledoux constant. Inversions of solar or stellar rotation profiles rely on this first order approximation (e.g. [26], [27], [28]). At more rapid rotation rates, one needs to include higher order effects of rotation (e.g. [29], [30]), and ultimately apply a 2D approach (e.g. [31], [32]). In what follows, we will describe the effects of rapid rotation on stellar pulsations, as based on the latest 2D numerical simulations.

\subsection{Gravito-inertial modes}

At the lower end of the frequency spectrum in non-rotating stars, there are gravity (or g-) modes for which the restoring force is buoyancy. When the star is rotating, buoyancy combines with the Coriolis force, thereby leading to gravito-inertial modes. The type of stars which display gravito-inertial modes are $\gamma$ Dor stars, SPBs, and Be stars. If the mode owes its existence to the Coriolis force, then it is known as an inertial mode. An extensive literature on inertial modes, and singular gravito-inertial modes exist (e.g. [33], [34], [35], [36], [37]), but in what follows, we will focus on modes which become classical g-modes in the non-rotating limit.

According to Tassoul's asymptotic formula [38], gravity modes in non-rotating stars are evenly spaced in period, for a given $\ell$ value. When the star is rotating, the period spacing also depends on $m$ and on the ratio $\eta=\frac{2 \Omega}{\omega}$ which characterises the effects of the Coriolis force on the pulsation mode, where $\Omega$ is the rotation rate and $\omega$ the pulsation frequency. Such a relation was first established using the traditional approximation ([39], [40]) and has been subsequently confirmed through full 2D calculations, although the centrifugal deformation slightly affects some of the modes [41].

In terms of mode geometry, it is extremely important to distinguish between sub-inertial modes, for which $\omega<2 \Omega$, and super-inertial modes, where $\omega>2 \Omega$. Indeed, in the sub-inertial regime, modes are confined to an equatorial region thanks to critical surfaces, as described in [42] and [36]. In the superinertial regime, gravito-inertial modes tend to behave like their non-rotating counterparts, except for one notable exception - rosette modes. Such modes where discovered by [41] using a 2D numerical approach, and have subsequently been studied in detail by [43], [44], [45], [46] using a variety of techniques.

\subsection{Acoustic modes}

At the upper end of the frequency spectrum, there are acoustic (or p-) modes for which the restoring force is pressure. Given that these modes tend to be located in the outer portions of the star, they are 
most affected by centrifugal deformation, which is greatest in such regions. One way of characterising the effects of the centrifugal deformation is by taking the ratio of the characteristic length scale of the deformation, which is roughly proportional to $\Omega^{2}$, to the mode's wavelength, which is proportional to $\omega^{-1}$. Hence, high frequency modes are more affected than low frequency modes, due to their smaller wavelength. $\delta$ Scuti and $\beta$ Cephei stars oscillate in the acoustic domain and typically tend to be rapid rotators.

At rapid rotation rates, the acoustic modes subdivide into different classes of modes, each with their own characteristic geometry and independent frequency organisation. This was first shown in polytropic models by [47], [48] using 2D numerical simulations and ray dynamics and was then extended to more realistic models using 2D numerical simulations by [49].

Among these different classes, island modes are the most important, since they are the most visible of the regular (non-chaotic) modes. These modes are the rotating counterpart to modes with few nodes from one pole to another, i.e. with a small $\ell-|m|$ value. Their geometric structure closely follows a 2-period ray orbit and is characterised by new quantum numbers as illustrated in Fig. 1. Using these quantum numbers, it is possible to obtain a new asymptotic formula which describes the frequencies of these modes, the coefficients of which are related to travel time integrals (see [47], [48], [50] and [51]). Of particular interest is the frequency spacing, $\Delta_{\tilde{n}}$, between modes with consecutive $\tilde{n}$ values. This spacing is, in fact, half the large separation, and roughly scales with the square-root of the stellar mean density, even a rapid rotation rates [52].

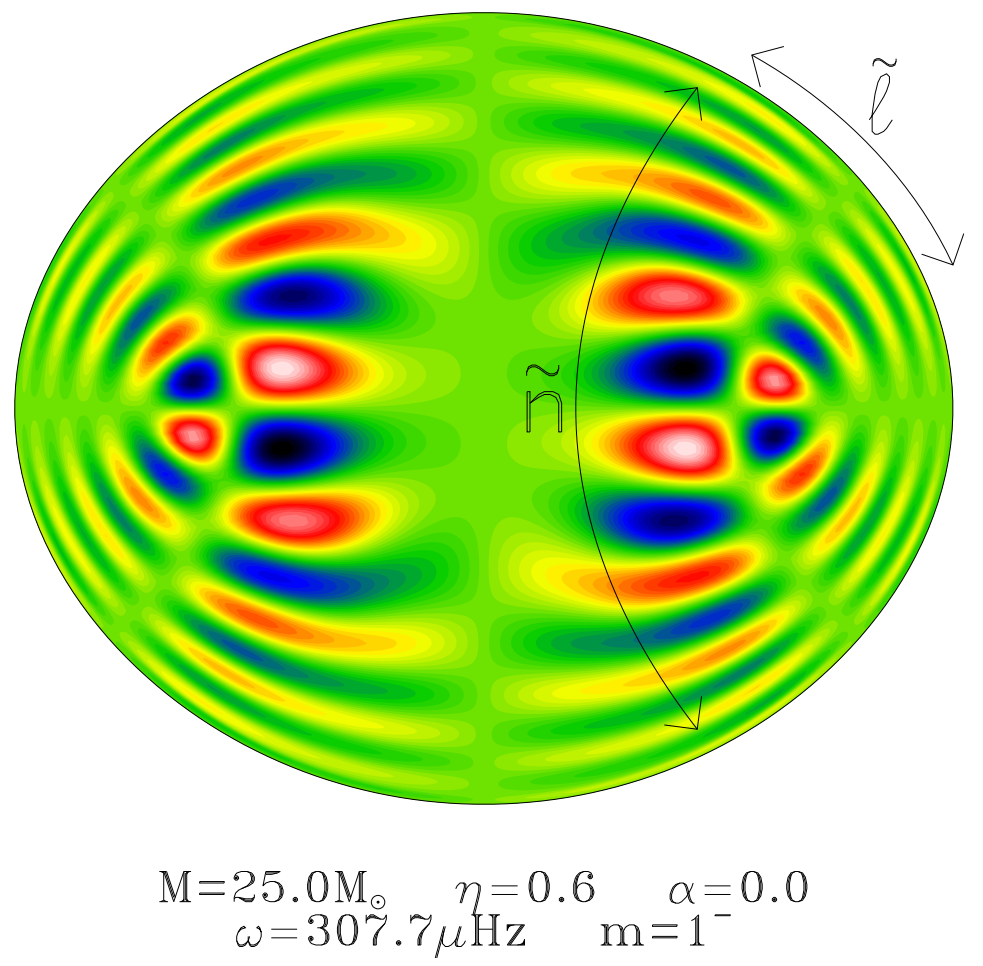

Fig. 1. Island mode in a model based on the Self-Consistent Field method [53]. New quantum numbers are indicated by the arrows. 


\subsection{Mixed modes}

Mixed modes combine the characteristics of acoustic and gravity modes, and occur when there are avoided crossings between these modes. Such modes typically occur in evolved stars, but can also be found in unevolved rapidly rotating stars. Indeed, gravito-inertial modes are hardly affected by centrifugal deformation and hence tend to scale as $\sqrt{G M / R_{\mathrm{pol}}^{3}}$, where $R_{\mathrm{pol}}$ is the polar radius. In contrast, acoustic modes and the characteristic spacing $\Delta_{\tilde{n}}$ are roughly proportional to $\sqrt{G M / V}$, where $V$ is the volume of the star. As a result, the frequency domain of acoustic modes decreases compared with that of gravito-inertial modes, thereby causing the two to overlap. This favours avoided crossings and hence mixed modes, especially given that rotation allows coupling between modes with different harmonic degrees.

In evolved stars, rotation may play an important role in mixed modes. Indeed, as shown by [54], rotation affects different members of a given rotation multiplet differently, even at relatively small rotation rates. This is because the avoided crossings which produce the mixed modes occur at slightly different values of the rotation rate for different members of the multiplet. As a result, the ratio of the $\mathrm{p}$ - and g-mode contributions will be different, thereby leading to different mode inertias, and the frequencies will no longer be evenly spaced.

\section{Asteroseismology}

Having described the effects of rapid rotation on stellar pulsations, we now turn our attention to the asteroseismic inferences which can be drawn from observed stellar pulsations. In what follows, we will distinguish between average or global asteroseismology, which focuses on the general characteristics of the pulsation spectrum, and detailed or "boutique" asteroseismology, which relies on identifying individual modes.

\subsection{Global asteroseismology}

As was done above, we first start with the lower end of the frequency spectrum. Recent observations have shown that the pulsation frequencies of gravito-inertial modes in a number of Be stars tend to clump together. As explained in various publications (e.g. [55], [56], [57]), the co-rotating frequencies of the pulsation modes are likely to be much smaller than the rotation rate. When viewed from an inertial frame, these frequencies are Doppler shifted by $m \Omega$ as described above, thereby leading to separate clumps for each azimuthal order. Accordingly, the frequency differences between these clumps provide an asteroseismic measure of the rotation rate. However, a recent publication, [58], showed discrepancies between this seismic rotation rate and more classical measurements of $\Omega$ based on spectroscopy.

At higher frequencies, recent studies of p-modes in rapidly rotating $\delta$ Scuti stars have found recurrent frequency spacings using histograms of frequency differences or Fourier transforms of frequency spectra ([59], [60], [61], [62], [63], [64]). Similar studies have also been carried out using theoretical pulsation spectra based on realistic mode visibilities ([65], [66]) and have shown that in favourable cases, such recurrent spacings could correspond to the large frequency spacing or half its value (i.e. $\Delta_{\tilde{n}}$, or to a multiple of the rotation rate. Interpreting these spacings as the large separation, [62] then went on to constrain the mean density of a $\delta$ Scuti star observed with CoRoT.

\subsection{Detailed asteroseismology}

In order to obtain tighter constraints on stellar properties, one needs to carry out detailed asteroseismology. However, up until now, it has proven to be very difficult to correctly match observed frequencies with theoretical modes, i.e. identify modes, in rapidly rotating stars due to the lack of simple frequency 
patterns, as is very well illustrated in Fig. 5 of [67]. In order to overcome these difficulties, one can envisage various approaches. One can still try to look for the more complicated patterns through the use of Echelle diagrams ([62], [64], [68]) or by matching an asymptotic formula to the frequencies [69]. The latter approach will however be thrown off by the presence of chaotic modes which follow their own frequency distribution [48]. Another possibility is to apply observational mode identification techniques based on multi-colour photometry or spectroscopy. Such techniques have already been applied in slowly rotating stars, but they need to be adapted to more rapid rotation.

In the photometric approach, one typically looks at the amplitude ratios or phase differences of a given pulsation mode in different photometric bands. The advantage of this approach is that the intrinsic mode amplitude factors out, thereby leaving information which is only based on mode geometry. As opposed to the non-rotating case, the amplitude ratios and phase differences depend both on the azimuthal order and on the inclination (e.g. [70], [71]). However, at a given inclination, similar amplitude ratios are obtained for modes with the same $\ell$ and $m$ but different radial orders [72]. This is due to the similar surface geometric structure of these modes, as expected from asymptotic theory [51]. Accordingly, by grouping together modes with similar amplitude ratios, one can find families of modes with similar quantum numbers, thereby constraining mode identification and characteristic spacings such as the large frequency separation or the rotation rate [66].

The spectroscopic approach consists in obtaining a time series of high resolution spectra of a pulsating star to see how the absorption lines vary over time as a result of Doppler shifts from the oscillatory motions, and comparing this to theoretical predictions. Such an approach cannot currently be carried out from space and requires the use of ground based-telescopes, preferably in a network such as SONG. Previous and current spectroscopic observations of rapidly rotating pulsating stars include those described in [73], [74], [75]. Various studies have focused on obtaining theoretical predictions in a rapidly rotating context ([76], [77], [78]) but more work is needed before these effects are incorporated into mode identification tools such as FAMIAS [79].

An important ingredient in predicting theoretical photometric and spectroscopic signatures of pulsation modes is the variations of effective temperature. However, this quantity can only be calculated through non-adiabatic pulsation calculations. Currently, the only studies that include these effects in rapidly rotating stars are those based on [80], which use models based on a Chandrasekhar expansion and focus on low frequency modes, and ongoing work which involves using the TOP pulsation code ([31], [49]) with models from the ESTER code, an approach which is applicable to all modes. Figure 2 shows a 2D excitation map as well as some 1D work integrals of stable and unstable acoustic islandmodes based on these latest calculations. An added benefit of non-adiabatic calculations is knowing which modes are unstable, which could provide additional constraints on the mode identification.

\section{Conclusion}

Rapid rotation plays a major role in the structure, evolution, and pulsations of massive and intermediate mass stars. Given that these stars are important for many domains of astrophysics, it is essential to understand the impact of rapid rotation. Recent theoretical developments are leading to more and more realistic models and a better understanding of their pulsation modes. Nonetheless, many questions remain unanswered, especially in the light of new and spectacular observations. Progress has been made in interpreting the general characteristics of observed pulsation spectra, but more work is needed in order to fully exploit the excellent data from current (MOST, CoRoT, Kepler, Brite) and future missions (TESS, PLATO).

DRR is funded by the European Community's Seventh Framework Programme (FP7/2007-2013) under grant agreement no. 312844 (SPACEINN), which is gratefully acknowledged. Funding for the Stellar Astrophysics Centre is provided by The Danish National Research Foundation (grant agreement No.: DNRF106). 

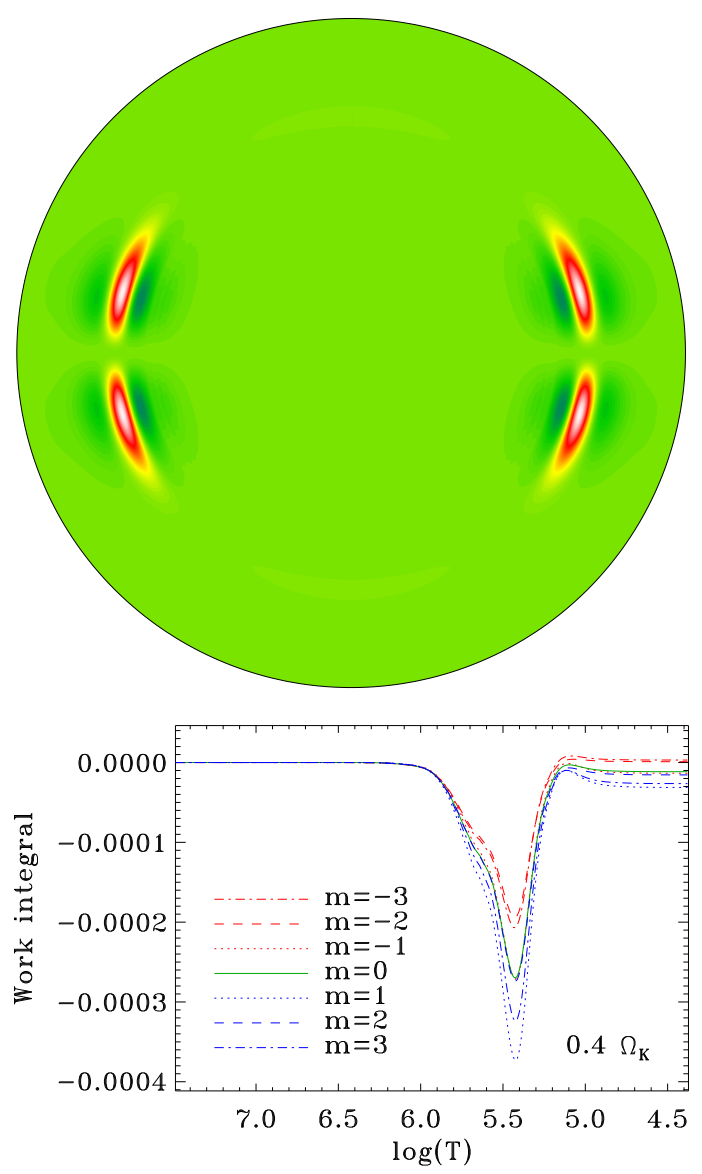

Fig. 2. (Upper panel) Two dimensional map of regions which excite (red/white) or damp (blue/black) a given island mode in an ESTER model. The radial coordinate in this plot is $\log T$ which stretches out the near surface regions where most of the excitation and damping takes places. (Lower panel) Work integrals for a multiplet of modes at $\Omega=0.4 \Omega_{\mathrm{K}}$. As can be seen, some of the members of this multiplet are stabilised by rotation.

\section{References}

1. F. Royer, On the Rotation of A-Type Stars, in The Rotation of Sun and Stars, edited by J.P. Rozelot, C. Neiner (2009), Vol. 765 of Lecture Notes in Physics, Berlin Springer Verlag, pp. 207-230

2. A. Domiciano de Souza, P. Kervella, S. Jankov, L. Abe, F. Vakili, E. di Folco, F. Paresce, A\&A 407, L47 (2003)

3. P. Kervella, A. Domiciano de Souza, A\&A 453, 1059 (2006)

4. A. Domiciano de Souza, M. Hadjara, F. Vakili, P. Bendjoya, F. Millour, L. Abe, A.C. Carciofi, D.M. Faes, P. Kervella, S. Lagarde et al., A\&A 545, A130 (2012)

5. D.M. Peterson, C.A. Hummel, T.A. Pauls, J.T. Armstrong, J.A. Benson, G.C. Gilbreath, R.B. Hindsley, D.J. Hutter, K.J. Johnston, D. Mozurkewich et al., ApJ 636, 1087 (2006)

6. J.D. Monnier, M. Zhao, E. Pedretti, N. Thureau, M. Ireland, P. Muirhead, J.P. Berger, R. MillanGabet, G. Van Belle, T. ten Brummelaar et al., Science 317, 342 (2007), arXiv: 0706.0867

7. X. Che, J.D. Monnier, M. Zhao, E. Pedretti, N. Thureau, A. Mérand, T. ten Brummelaar, H. McAlister, S.T. Ridgway, N. Turner et al., ApJ 732, 68 (2011)

8. J.C. Suárez, E. Michel, F. Pérez Hernández, Y. Lebreton, Z.P. Li, L. Fox Machado, A\&A 390, 523 (2002) 
9. S.J.A.J. Salmon, J. Montalbán, D.R. Reese, M.A. Dupret, P. Eggenberger, A\&A 569, A18 (2014)

10. H. von Zeipel, MNRAS 84, 665 (1924)

11. L.B. Lucy, Zeitschrift für Astrophysic 65, 89 (1967)

12. F. Espinosa Lara, M. Rieutord, A\&A 533, A43 (2011)

13. M. Rieutord, B. Dintrans, F. Lignières, T. Corbard, B. Pichon, The ESTER project, in SF2A-2005: Semaine de l'Astrophysique Francaise (2005), pp. 759-762

14. M. Rieutord, F. Espinosa Lara, Communications in Asteroseismology 158, 99 (2009)

15. F. Espinosa Lara, M. Rieutord, A\&A 552, A35 (2013)

16. G. Meynet, A. Maeder, A\&A 361, 101 (2000), astro-ph/0006404

17. J.P. Zahn, A\&A 265, 115 (1992)

18. G. Meynet, A. Maeder, Rotation and Mixing in Massive Stars: Principles and Uncertainties, in The Nature and Evolution of Disks Around Hot Stars, edited by R. Ignace, K.G. Gayley (2005), Vol. 337 of Astronomical Society of the Pacific Conference Series, pp. 15-+, astro-ph/0409722

19. I. Hunter, I. Brott, D.J. Lennon, N. Langer, P.L. Dufton, C. Trundle, S.J. Smartt, A. de Koter, C.J. Evans, R.S.I. Ryans, ApJ 676, L29 (2008)

20. I. Brott, C.J. Evans, I. Hunter, A. de Koter, N. Langer, P.L. Dufton, M. Cantiello, C. Trundle, D.J. Lennon, S.E. de Mink et al., A\&A 530, A116 (2011)

21. C. Aerts, G. Molenberghs, M.G. Kenward, C. Neiner, ApJ 781, 88 (2014)

22. F. Espinosa Lara, M. Rieutord, A\&A 470, 1013 (2007)

23. A. Maeder, C. Georgy, G. Meynet, A\&A 479, L37 (2008)

24. J. Wang, C. Liang, M.S. Miesch, Journal of Computational Physics (in preparation)

25. P. Ledoux, ApJ 114, 373 (1951)

26. J. Schou, H.M. Antia, S. Basu, R.S. Bogart, R.I. Bush, S.M. Chitre, J. Christensen-Dalsgaard, M.P. di Mauro, W.A. Dziembowski, A. Eff-Darwich et al., ApJ 505, 390 (1998)

27. S. Deheuvels, R.A. García, W.J. Chaplin, S. Basu, H.M. Antia, T. Appourchaux, O. Benomar, G.R. Davies, Y. Elsworth, L. Gizon et al., ApJ 756, 19 (2012)

28. D.W. Kurtz, H. Saio, M. Takata, H. Shibahashi, S.J. Murphy, T. Sekii, MNRAS 444, 102 (2014), See also these proceedings

29. H. Saio, ApJ 244, 299 (1981)

30. F. Soufi, M.J. Goupil, W.A. Dziembowski, A\&A 334, 911 (1998)

31. D. Reese, F. Lignières, M. Rieutord, A\&A 455, 621 (2006), astro-ph/0605503

32. J. Ballot, F. Lignières, D.R. Reese, M. Rieutord, A\&A 518, A30 (2010)

33. J. Papaloizou, J.E. Pringle, MNRAS 182, 423 (1978)

34. U. Lee, MNRAS 365, 677 (2006)

35. M. Rieutord, B. Georgeot, L. Valdettaro, Physical Review Letters 85, 4277 (2000)

36. B. Dintrans, M. Rieutord, A\&A 354, 86 (2000)

37. G. Mirouh, Gravito-inertial waves in a differentially rotating spherical shell, in These proceedings, edited by Ballot, J. and García, R. A. (2014)

38. M. Tassoul, ApJS 43, 469 (1980)

39. G. Berthomieu, G. Gonczi, P. Graff, J. Provost, A. Rocca, A\&A 70, 597 (1978)

40. U. Lee, H. Saio, MNRAS 224, 513 (1987)

41. J. Ballot, F. Lignières, V. Prat, D.R. Reese, M. Rieutord, 2D Computations of g-modes in Fast Rotating Stars, in Progress in Solar/Stellar Physics with Helio- and Asteroseismology, edited by H. Shibahashi, M. Takata, A.E. Lynas-Gray (2012), Vol. 462 of Astronomical Society of the Pacific Conference Series, p. 389

42. B. Dintrans, M. Rieutord, L. Valdettaro, Journal of Fluid Mechanics 398, 271 (1999)

43. M. Takata, H. Saio, Publ. of the Astronomical Society of Japan 65, 68 (2013)

44. H. Saio, M. Takata, Publ. of the Astronomical Society of Japan 66, 58 (2014)

45. M. Takata, Publ. of the Astronomical Society of Japan 66, 80 (2014)

46. M. Takata, H. Saio, Rosette modes of oscillations in rotating stars as a new aspect of rotationpulsation interaction, in These proceedings, edited by Ballot, J. and García, R. A. (2014)

47. F. Lignières, B. Georgeot, PRE 78, 016215 (2008), 0803 . 1737

48. F. Lignières, B. Georgeot, A\&A 500, 1173 (2009), 0903.1768

49. D.R. Reese, K.B. MacGregor, S. Jackson, A. Skumanich, T.S. Metcalfe, A\&A 506, 189 (2009) 
50. M. Pasek, B. Georgeot, F. Lignières, D.R. Reese, Physical Review Letters 107, 121101 (2011)

51. M. Pasek, F. Lignières, B. Georgeot, D.R. Reese, A\&A 546, A11 (2012)

52. D. Reese, F. Lignières, M. Rieutord, A\&A 481, 449 (2008), 0801.4630

53. K.B. MacGregor, S. Jackson, A. Skumanich, T.S. Metcalfe, ApJ 663, 560 (2007), arXiv:0704.1275

54. R.M. Ouazzani, M.J. Goupil, M.A. Dupret, J.P. Marques, A\&A 554, A80 (2013)

55. G.A.H. Walker, R. Kuschnig, J.M. Matthews, C. Cameron, H. Saio, U. Lee, E. Kambe, S. Masuda, D.B. Guenther, A.F.J. Moffat et al., ApJ 635, L77 (2005)

56. H. Saio, C. Cameron, R. Kuschnig, G.A.H. Walker, J.M. Matthews, J.F. Rowe, U. Lee, D. Huber, W.W. Weiss, D.B. Guenther et al., ApJ 654, 544 (2007)

57. C. Cameron, H. Saio, R. Kuschnig, G.A.H. Walker, J.M. Matthews, D.B. Guenther, A.F.J. Moffat, S.M. Rucinski, D. Sasselov, W.W. Weiss, ApJ 685, 489 (2008)

58. T. Semaan, J. Gutiérrez-Soto, Y. Frémat, A.M. Hubert, C. Martayan, J. Zorec, A Pulsational Study of a Sample of CoRoT Faint Be Stars, in Stellar Pulsations: Impact of New Instrumentation and New Insights, edited by J.C. Suárez, R. Garrido, L.A. Balona, J. Christensen-Dalsgaard (2013), Vol. 31 of Advances in Solid State Physics, p. 261

59. M. Breger, P. Lenz, A.A. Pamyatnykh, MNRAS 396, 291 (2009)

60. A. García Hernández, A. Moya, E. Michel, R. Garrido, J.C. Suárez, E. Rodríguez, P.J. Amado, S. Martín-Ruiz, A. Rolland, E. Poretti et al., A\&A 506, 79 (2009)

61. L. Mantegazza, E. Poretti, E. Michel, M. Rainer, F. Baudin, A. García Hernández, T. Semaan, M. Alvarez, P.J. Amado, R. Garrido et al., A\&A 542, A24 (2012)

62. A. García Hernández, A. Moya, E. Michel, J.C. Suárez, E. Poretti, S. Martín-Ruíz, P.J. Amado, R. Garrido, E. Rodríguez, M. Rainer et al., A\&A 559, A63 (2013)

63. J.C. Suárez, A. García Hernández, A. Moya, C. Rodrigo, E. Solano, R. Garrido, J.R. Rodón, A\&A 563, A7 (2014)

64. A. García Hernández, F. Lignières, L. Balona, D.R. Reese, M.J.P.F.G. Monteiro, J.C. Suárez, J. Ballot, Patterns, an efficient way to analyse the p-mode content in rapidly rotating stars, in These proceedings, edited by Ballot, J. and García, R. A. (2014)

65. F. Lignières, B. Georgeot, J. Ballot, Astronomische Nachrichten 331, 1053 (2010)

66. D.R. Reese, F. Lignières, J. Ballot, M.A. Dupret, C. Barban, C. van 't Veer-Menneret, K.B. MacGregor, A\&A (submitted)

67. R.G. Deupree, D. Castañeda, F. Peña, C.I. Short, ApJ 753, 20 (2012)

68. T. Bedding, S. Murphy, D. Stello, Unlocking the secrets of gamma Doradus and delta Scuti stars using echelle diagrams, in These proceedings, edited by Ballot, J. and García, R. A. (2014)

69. D.R. Reese, M.J. Thompson, K.B. MacGregor, S. Jackson, A. Skumanich, T.S. Metcalfe, A\&A 506, 183 (2009)

70. R.H.D. Townsend, MNRAS 343, 125 (2003)

71. J. Daszynska-Daszkiewicz, W.A. Dziembowski, A.A. Pamyatnykh, Acta Astronomica 57, 11 (2007)

72. D.R. Reese, V. Prat, C. Barban, C. van 't Veer-Menneret, K.B. MacGregor, A\&A 550, A77 (2013)

73. J.H. Telting, C. Schrijvers, A\&A 339, 150 (1998)

74. E. Poretti, E. Michel, R. Garrido, L. Lefèvre, L. Mantegazza, M. Rainer, E. Rodríguez, K. Uytterhoeven, P.J. Amado, S. Martín-Ruiz et al., A\&A 506, 85 (2009)

75. N. Themeß1, Frequency analysis and spectroscopic mode identification of the delta Scuti star 4 $C V n$, in These proceedings, edited by Ballot, J. and García, R. A. (2014)

76. U. Lee, H. Saio, ApJ 349, 570 (1990)

77. M.J. Clement, Pulsation in Rapidly Rotating Stars, in IAU Symp. 162: Pulsation; Rotation; and Mass Loss in Early-Type Stars, edited by L.A. Balona, H.F. Henrichs, J.M. Le Contel (1994), pp. 117-+

78. R.H.D. Townsend, MNRAS 284, 839 (1997)

79. W. Zima, Communications in Asteroseismology 155, 17 (2008)

80. U. Lee, I. Baraffe, A\&A 301, 419 (1995) 